\title{
Neutrophils and their Fc $\gamma$ receptors are essential in a mouse model of transfusion-related acute lung injury
}

\author{
Mark R. Looney, ${ }^{1,2}$ Xiao Su, ${ }^{1}$ Jessica A. Van Ziffle, ${ }^{3}$ Clifford A. Lowell, ${ }^{3}$ and Michael A. Matthay ${ }^{1,2,4}$ \\ ${ }^{1}$ Cardiovascular Research Institute, ${ }^{2}$ Department of Medicine, ${ }^{3}$ Department of Laboratory Medicine, and \\ ${ }^{4}$ Department of Anesthesia, UCSF, San Francisco, California, USA.
}

\begin{abstract}
Transfusion-related acute lung injury (TRALI) is the most common cause of transfusion-related mortality. To explore the pathogenesis of TRALI, we developed an in vivo mouse model based on the passive transfusion of an MHC class I (MHC I) $\mathrm{mAb}\left(\mathrm{H}_{2} \mathrm{~K}^{\mathrm{d}}\right)$ to mice with the cognate antigen. Transfusion of the MHC I $\mathrm{mAb}$ to $\mathrm{BALB} / \mathrm{c}$ mice produced acute lung injury with increased excess lung water, increased lung vascular and lung epithelial permeability to protein, and decreased alveolar fluid clearance. There was $50 \%$ mortality at a 2-hour time point after $\mathrm{Ab}$ administration. Pulmonary histology and immunohistochemistry revealed prominent neutrophil sequestration in the lung microvasculature that occurred concomitantly with acute peripheral blood neutropenia, all within 2 hours of administration of the $\mathrm{mAb}$. Depletion of neutrophils by injection of anti-granulocyte $\mathrm{mAb} \mathrm{Gr}-1$ protected mice from lung injury following MHC I mAb challenge. $F c R \gamma^{-/-}$mice were resistant to MHC I mAb-induced lung injury, while adoptive transfer of wild-type neutrophils into the $F c R \gamma^{-/-}$animals restored lung injury following MHC I mAb challenge. In conclusion, in a clinically relevant in vivo mouse model of TRALI using an MHC I mAb, the mechanism of lung injury was dependent on neutrophils and their Fc $\gamma$ receptors.
\end{abstract}

\section{Introduction}

Transfusion-related acute lung injury (TRALI) is defined as acute lung injury (ALI) that develops during or within 6 hours after transfusion of a plasma-containing blood product (1). TRALI has a reported incidence of 1 in 5,000 transfused blood products or 1 in 600 persons transfused, but it is generally accepted that TRALI is underdiagnosed and underreported (1-3). In recent years, TRALI has emerged as the leading cause of transfusionrelated fatalities reported to the $\operatorname{FDA}(1,4)$. There is an urgent need to better understand the pathogenesis of this serious complication of transfusion therapy.

TRALI is thought to develop from the passive transfusion of donor biologic response modifiers to recipients. Donor HLA I and II Abs and anti-neutrophil Abs have been widely reported in the literature dating back to the first TRALI case series in 1985 (2, 5). In fact, HLA I is the Ab most frequently associated with fatal cases of TRALI (6). Other investigators have focused on the possible contribution of biologically active lipids that can accumulate in stored cellular blood products that may prime and activate neutrophils (7).

The existing animal models of TRALI, while proving the principle of $\mathrm{Ab}$ - and lipid-based lung injury from transfusions, are limited by ex vivo conditions and cross-species transfusions (8-11). An isolated, perfused rabbit lung model of TRALI used human neutrophils, a human anti-neutrophil $\mathrm{Ab}$, and rabbit complement to produce lung injury (8). In another ex vivo model, rat lungs were perfused with plasma from outdated human packed red blood cells

Nonstandard abbreviations used: ALI, acute lung injury; ARDS, acute respiratory distress syndrome; BAL, bronchoalveolar lavage; BALB/K mouse, BALB/c congenic mouse; EVPE, extravascular plasma equivalents; MHC I, MHC class I; TRALI, transfusion-related acute lung injury.

Conflict of interest: The authors have declared that no conflict of interest exists. Citation for this article: J. Clin. Invest. 116:1615-1623 (2006). doi:10.1172/JCI27238 and human whole blood and apheresis platelets as well as the lipid fraction from these blood units to produce lung injury $(10,11)$.

Questions related to the pathogenic mechanisms in TRALI can be better addressed using in vivo mouse models, which afford the opportunity to use genetically manipulated animals. Therefore, for these studies, we developed an in vivo mouse model of TRALI based on the passive transfusion of an MHC class I (MHC I) mAb to mice. By developing this clinically relevant mouse model of TRALI, we have found a critical role for neutrophil Fcy receptors in mediating ALI.

\section{Results}

MHC I mAb produces ALI in mice. Wild-type BALB/c mice were selected to receive the MHC I $\mathrm{mAb}\left(\operatorname{anti}-\mathrm{H} 2 \mathrm{~K}^{\mathrm{d}}\right)$, since this mouse strain expresses $\mathrm{H} 2 \mathrm{~K}^{\mathrm{d}}$. BALB/c congenic $(\mathrm{BALB} / \mathrm{K})$ mice, which have a $\mathrm{H} 2 \mathrm{~K}^{\mathrm{k}}$ MHC I locus, were used as controls. An additional control

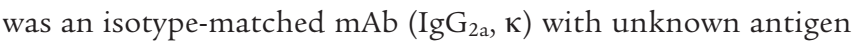
specificity. All mice received an equal-volume i.v. injection. MHC I $\mathrm{mAb}$-treated BALB/c mice developed a sharp increase in lung water at 2 hours after i.v. challenge (Figure 1A). In contrast, the $\mathrm{BALB} / \mathrm{K}$ animals failed to show evidence of pulmonary vascular leak following injection of anti-H $2 \mathrm{~K}^{\mathrm{d}}$, confirming that the disease was caused by recognition of the d allotype of MHC I protein in normal $\mathrm{BALB} / \mathrm{c}$ mice. In addition, the isotype-matched $\mathrm{mAb}$ given to $\mathrm{BALB} / \mathrm{c}$ mice did not produce an increase in lung water. The increase in extravascular lung water in BALB/c MHC I mAb-treated mice observed in Figure $1 \mathrm{~A}$ was matched by the increase in lung vascular permeability to protein ( ${ }^{125} \mathrm{I}$-labeled albumin; Figure $\left.1 \mathrm{~B}\right)$. An MHC I mAb dose-response curve was determined, and a dose of $4.5 \mathrm{mg} / \mathrm{kg}$ produced the most reproducible lung injury (data not shown). Since the isotype-matched $\mathrm{mAb}$-treated mice and the $\mathrm{BALB} / \mathrm{K}$ mice challenged with MHC I mAb develop no lung injury, in other experiments in this study, PBS delivered to wild-type $\mathrm{BALB} / \mathrm{c}$ mice was used as the control. 


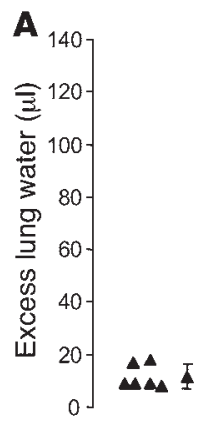

$80 \bar{s}$

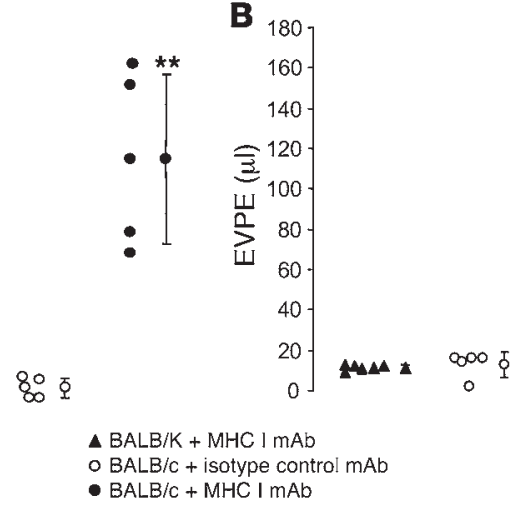

Figure 1

MHC I mAb produces ALI in BALB/c mice. Gravimetric excess lung water (A) and EVPE determined from i.v. ${ }^{125}$-labeled albumin protein leakage $(\mathbf{B})$ were increased in $B A L B / c$ mice $\left(H_{2} K^{d}\right)$ given $\mathrm{MHC}$ I mAb $(4.5 \mathrm{mg} / \mathrm{kg})$ but not in $\mathrm{BALB} / \mathrm{K}$ controls or in $\mathrm{BALB} / \mathrm{c}$ mice given an isotype-matched $A b\left(\operatorname{lgG}_{2 a}, \kappa\right)$. Each data point represents a single animal. ${ }^{* *} P<0.01$ compared with BALB/K $+\mathrm{MHC}$ I mAb and BALB/c + isotype control mAb.

MHC I mAb-treated mice have increased mortality. Death before the 2-hour end point of the experiments was observed in the MHC I $\mathrm{mAb}$-treated mice. There was approximately $50 \%$ mortality in the MHC I mAb-treated mice compared with PBS or isotype-matched $\mathrm{mAb}$ controls (Figure 2A). Figure 2, B and C, show that the MHC I $\mathrm{mAb}$-challenged mice that died before 2 hours had worse lung injury compared with the mice who survived the MHC I $\mathrm{mAb}$ challenge. We frequently observed frothy pulmonary edema from the mouth and nose of moribund mice.

MHC I mAb-treated mice have increased alveolar epithelial permeability, increased bronchoalveolar lavage total protein, and decreased alveolar fluid clearance. Having observed that the MHC I mAb produced ALI and induced $50 \%$ mortality, we next focused on the mechanisms of lung injury. From several animal (12) and human studies $(13,14)$, the importance of the alveolar epithelium to the development and resolution of lung injury has been appreciated. In experimental models there is often dissociation in the extent of injury to the lung capillary endothelium and the alveolar epithelium (15). In this MHC I mAb model of lung injury, both the lung endothelium (Figure 1B) and the alveolar epithelium were permeable to protein ( ${ }^{125}$ I-labeled albumin; Figure 3A). Using another index of permeability pulmonary edema, we measured the total protein concentration in the bronchoalveolar lavage (BAL) of mice challenged with MHC I mAb. Mice challenged with MHC I mAb had increased total protein concentrations in the airspaces compared with controls, reflecting increased protein flux across both the lung endothelium and the lung epithelium (Figure 3B). Next, using our in situ model of alveolar fluid clearance, we assessed the function of the alveolar epithelium in MHC I mAb-induced lung injury. PBS-treated mice had intact alveolar fluid clearance at 2 hours, consistent with previously published results $(16,17)$. However, the MHC I mAb-treated mice had impaired alveolar fluid clearance compared with PBS controls (Figure 3C). Thus the lung edema formation shown in Figure 1A reflects both an increased lung vascular and lung epithelial permeability to protein and a decreased capacity to remove edema fluid from the alveoli.
MHC I mAb-challenged mice develop severe pulmonary neutrophil sequestration and peripheral blood neutropenia. Histologic examination of the lungs from MHC I mAb-treated mice revealed obvious septal thickening and severe inflammatory infiltrates within 2 hours after $\mathrm{mAb}$ challenge (Figure 4, compare A and B). Most of these inflammatory cells appeared to be granulocytes, many of which plugged branching microvascular vessels within the pulmonary parenchyma (Figure 4C). Animals with obvious clinical evidence of pulmonary edema manifested histologic evidence of intra-alveolar proteinaceous fluid (Figure 4D).

To further clarify the neutrophil sequestration in MHC I mAbtreated mice, we performed immunohistochemistry using the neutrophil-specific mAb Gr-1. This immunohistochemistry confirmed extensive neutrophil sequestration in the lungs 2 hours following MHC I mAb challenge (Figure 5, A and B). Of note, there was only mild neutrophilia (0-5\%) in the BAL of MHC I mAb-challenged mice at the early time point of 2 hours (data not shown).

Peripheral blood neutropenia is commonly observed in TRALI patients, potentially due to pulmonary sequestration of circulating cells $(2,18)$. Similar to the human cases, we also observed acute neutropenia in the MHC I mAb-treated animals (Figure 5C). Thrombocytopenia has also been reported in some cases in the human TRALI literature (19-21), but none was detected in this experimental model (data not shown).

MHC I mAb-treated mice have a selective increase in plasma cytokines and chemokines. Multiplex cytokine analysis revealed significant differences between controls and MHC I mAb-treated mice in 3 cytokines. Two prototypic inflammatory cytokines, TNF- $\alpha$ and IL-6, and an antiinflammatory cytokine, IL-10, were elevated in MHC I mAb-treated mice (Figure 6A). Plasma levels of IFN- $\gamma$,
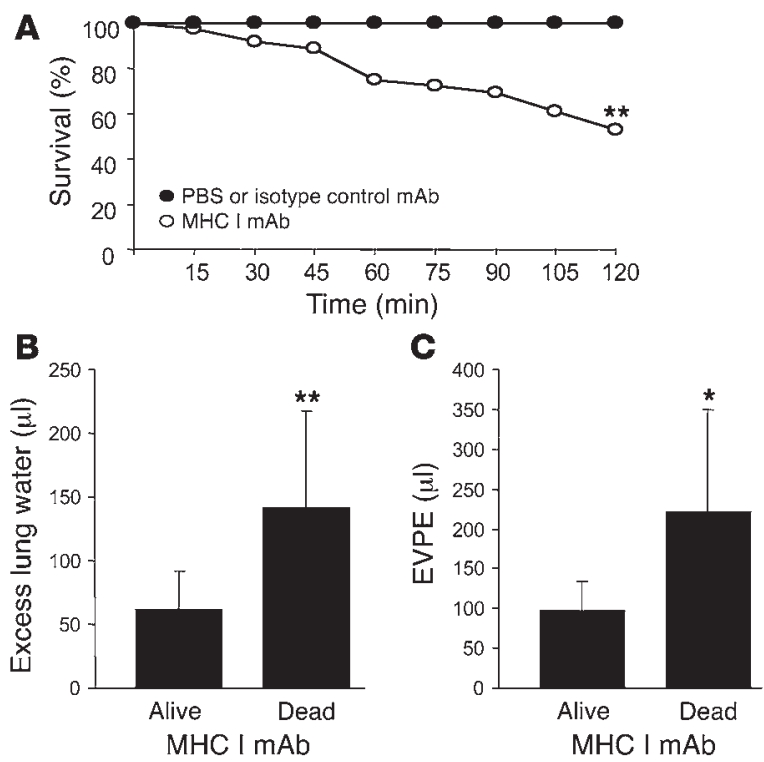

Figure 2

MHC I mAb produces mortality and increased lung injury in nonsurvivors versus survivors. (A) BALB/c mice given $\mathrm{MHC}$ I $\mathrm{mAb}(n=36)$ showed decreased survival at 2 hours compared with BALB/c mice given either isotype-matched mAb or PBS $(n=22)$. ${ }^{*} P<0.01, \chi^{2}$ test. The MHC I mAb-challenged mice that died before 2 hours $(n=15)$ had increased excess lung water (B) and increased EVPE (C) compared with $\mathrm{MHC}$ I mAb-challenged mice that survived the 2-hour experimental period $(n=15) .{ }^{* \star} P<0.01 ;{ }^{*} P<0.05$. 

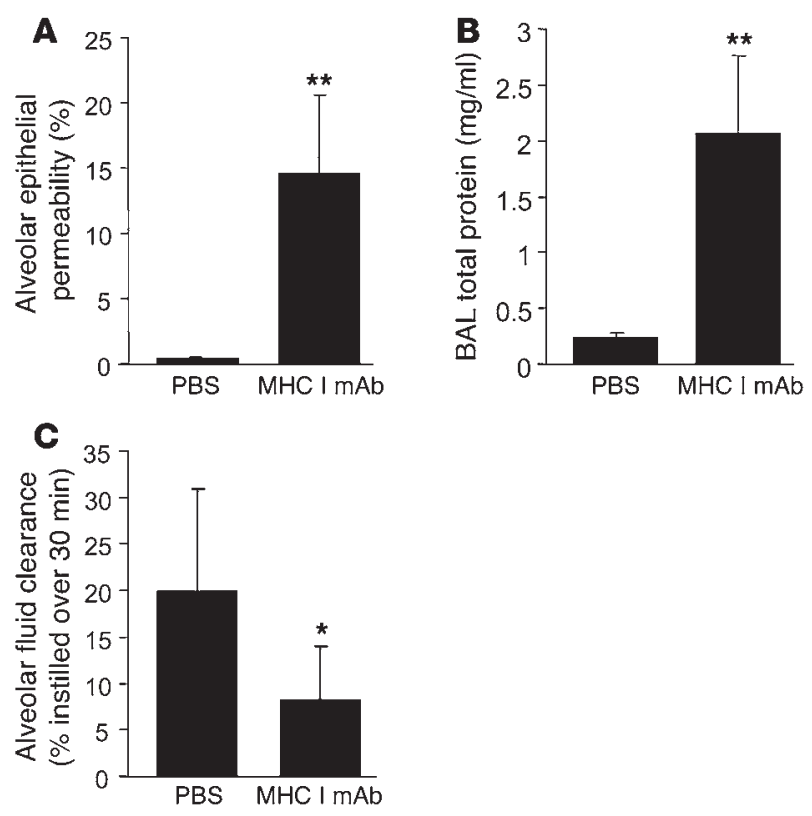

Figure 3

MHC I mAb produces increased alveolar epithelial permeability and decreased alveolar fluid clearance. (A and $\mathbf{B}$ ) Alveolar epithelial permeability to ${ }^{125}$ |-labeled albumin (A) and BAL total protein (B) in BALB/c mice given PBS $(n=6)$ or MHC I mAb $(n=10) .{ }^{* \star} P<0.01$. (C) In situ alveolar fluid clearance was measured over 30 minutes in mice treated with $\mathrm{MHC}$ I mAb $(n=7)$ or PBS $(n=6) .{ }^{*} P<0.05$.

GM-CSF, IL-1 $\beta$, IL-2, IL-4, IL-5, and IL-12 were not increased in MHC I mAb-treated mice compared with controls. Since there was histologic evidence of lung neutrophil sequestration in MHC I $\mathrm{mAb}$-challenged mice, plasma and BAL concentrations of the murine IL- 8 homologs, $\mathrm{KC}$ and macrophage inflammatory protein-2 (MIP-2), were measured. Both KC and MIP-2 levels were increased in the plasma (Figure 6B) and BAL (Figure 6C) of MHC I $\mathrm{mAb}$-challenged mice compared with controls.

Neutrophil depletion protects mice from MHCI $m A b$-induced ALI. Neutrophils are prominent cellular mediators in some animal models of ALI (22), and neutrophil depletion has been shown to be protective in models of lung injury such as acid injury (23) and hemorrhagic shock (24). Given the extensive neutrophil sequestration observed in the lungs of MHC I mAb-treated mice, we hypothesized that neutrophil depletion would protect animals from MHC I $\mathrm{mAb}$-induced lung injury. We used the Gr-1 mAb to selectively deplete mouse neutrophils, as has been previously described (25, $26)$. Within 24 hours, there was near complete neutrophil depletion in Gr-1 mAb-treated mice ( $50 \pm 35$ versus $783 \pm 394$ neutrophils $/ \mathrm{ml}$ for Gr-1 mAb and PBS groups, respectively) with preservation of lymphocyte, monocyte, and platelet counts and hemoglobin (data not shown). After confirming neutrophil depletion in the Gr-1 $\mathrm{mAb}$-treated mice, these animals were challenged with MHC I mAb or control. The Gr-1 mAb neutrophil-depleted mice were protected from lung injury, demonstrating lung water and lung vascular permeability values similar to control mice (Figure 7, A and B). We also used a less specific method of neutrophil depletion, i.p. vinblastine, which led to greater than $95 \%$ neutrophil depletion at 72 hours. Vinblastine-treated mice were also protected from lung edema formation and lung vascular permeability to protein when challenged with i.v. MHC I mAb (data not shown).
FcR $\gamma^{-1-}$ mice are protected from MHC I mAb-induced ALI, while adoptive transfer of wild-type and MHC I-deficient neutrophils restores lung injury in $\mathrm{FcR} \gamma^{-1-}$ animals following MHC I mAb challenge. To determine whether Ab-based TRALI involves specific immune recognition of endothelial-bound MHC Abs by neutrophils, we studied $F c R \gamma^{-/-}$ mice, which lack the $\gamma$ chain subunit of IgG and IgE receptors. $F c R \gamma^{-/-}$mice lack both the high-affinity FcyRI and low-affinity Fc $\gamma$ RIII receptors and have been shown to be refractory to stimulatory Fc $\gamma$ receptor signaling (27). FcR $\gamma^{-/-}$mice on the BALB/c genetic background were compared with age- and sex-matched wild-type BALB/c mice in the anti-MHC I mAb model. As shown in Figure 8, $F c R \gamma^{-/-}$mice were completely protected from MHC I $\mathrm{mAb}$-mediated lung injury compared with wild-type controls.

Since Fc $\gamma \mathrm{RI}$ and Fc $\mathrm{R}$ III receptors are present not only on neutrophils but also on lymphocytes, monocytes, mast cells, and dendritic cells, the specific contribution of the Fcy receptor on neutrophils cannot be inferred from the above experiments. To answer this question, we adoptively transferred (by i.v. injection) wild-type neutrophils $\left(5 \times 10^{6}\right.$ cells $)$ into $F c R \gamma^{-1-}$ mice, then immediately challenged these animals with anti-MHC I mAb. Injection of wildtype neutrophils into $\mathrm{FcR \gamma ^{-/- }}$ mice restored ALI following MHC I $\mathrm{mAb}$ delivery (Figure 8, A and B). Untreated animals showed no evidence of lung injury from the neutrophil transfusion (data not shown). Additionally, when $F c R \gamma^{-/-}$mice were reconstituted with MHC I-deficient neutrophils $\left(B 2 \mathrm{~m}^{-/}\right)$, lung injury was again restored with $\mathrm{MHC}$ I $\mathrm{mAb}$ challenge, which excludes direct neutrophil activation by binding of the MHC I mAb to the cell surface as a pathogenic event. In summary, these data demonstrate that Fcy receptors on neutrophils alone are responsible for generation of ALI in this model.

MHC I $m A b$ delivered i.v. binds diffusely to the microvasculature of the lung, liver, and kidney. Using Rag $2^{-/-}$mice $\left(\mathrm{H} 2 \mathrm{~K}^{\mathrm{d}+}\right)$, which lack endogenous Ig, we tracked the in vivo binding of i.v. MHC I mAb with immunohistochemistry. Of note, Rag2 ${ }^{-1-}$ mice challenged with MHC I mAb developed similar lung injury compared with wild-type

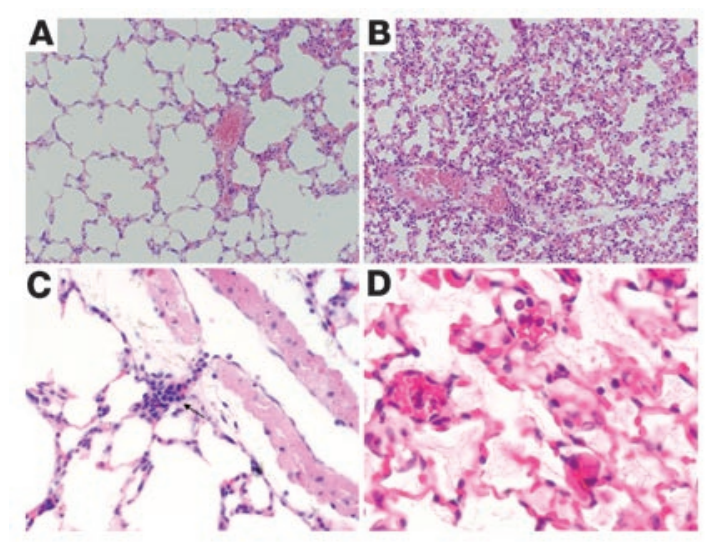

\section{Figure 4}

Lung histology from control and $\mathrm{MHC}$ I mAb-treated mice. (A and B) Low-power views of lungs from mice given either control (A) or $\mathrm{MHC}$ I $\mathrm{mAb}(\mathbf{B})$. In the MHC I mAb-treated mouse, there was increased intravascular neutrophils, septal thickening, and interstitial inflammation. (C) High-power view of lung from a mouse given MHC I mAb. Arrow indicates a branching vessel that is plugged with neutrophils. (D) $\mathrm{MHC}$ I mAb-treated mouse with intra-alveolar proteinaceous material. $\mathrm{H} \& \mathrm{E}$ staining. Magnification, $\times 200(\mathbf{A}$ and $\mathbf{B}) ; \times 400(\mathbf{C}$ and $\mathbf{D})$. 

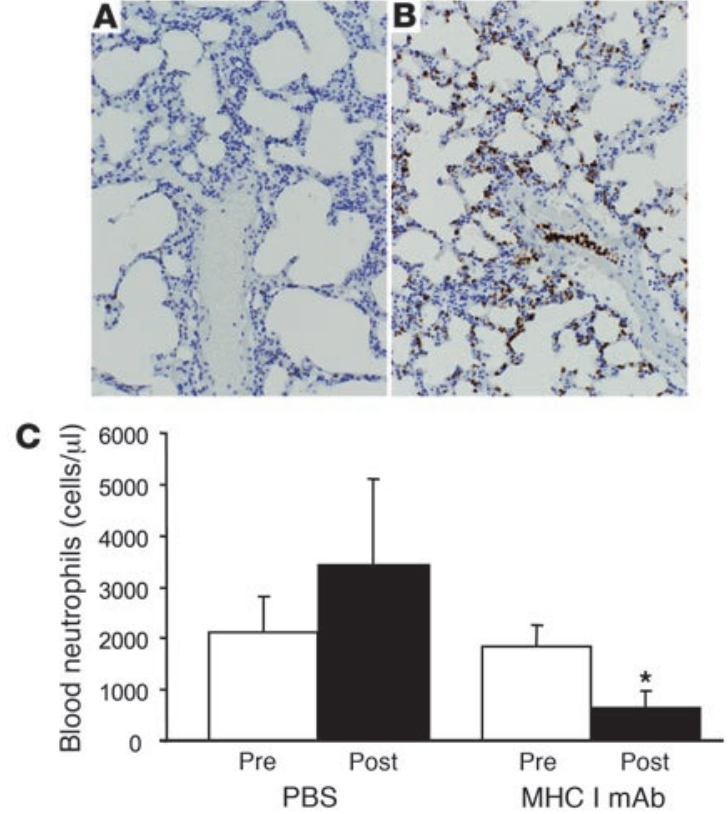

Figure 5

MHC I mAb-treated mice develop lung neutrophil sequestration and neutropenia. (A and B) Low-power view of lung from BALB/c mouse given either PBS (A) or MHC I mAb (B) and stained with Gr-1 antineutrophil $m A b$. Note the extensive neutrophil sequestration (brown color) in the MHC I mAb-treated mouse lung. Magnification, $\times 200$. (C) Neutrophil levels in the blood. Two hours after injection of either PBS or $\mathrm{MHC}$ I mAb compared with baseline preinjection levels. ${ }^{*} P<0.05$.

BALB/c controls (data not shown). As shown in Figure 9, the lung, liver, and kidney microvasculature all bound the i.v.-delivered $\mathrm{mAb}$. The lung, the first capillary bed encountered by the i.v. mAb, demonstrated especially dense staining compared with the downstream kidney and liver microvasculature. Interestingly, in preliminary experiments, modest damage to the kidney but not to the liver was detected at the 2-hour experimental end point (kidney wet wt/dry wt ratio, $3.42 \pm 0.38$ versus $2.62 \pm 0.28$ [mean $\pm \mathrm{SD}$ ] for $\mathrm{MHC}$ I mAb and isotype control $\mathrm{mAb}$, respectively; $P<0.01$ ).

MHC I $m A b$ binds to neutrophils but does not directly activate neutrophils. Because other animal models $(8,9)$ and multiple clinical studies $(2,3)$ have implicated anti-neutrophil Abs in the pathogenesis of TRALI, we investigated whether neutrophils bind to and/or are directly activated by MHC I mAb. Since neutrophils express MHC I antigen, it was not surprising that wild-type and $F c R \gamma^{-/-}$neutrophils bound MHC I $\mathrm{mAb}$, but MHC I-deficient neutrophils $\left(\mathrm{B}^{\left.\mathrm{m} \mathrm{m}^{-/-}\right)}\right.$did not (Figure 10A). Using a flow cytometry-based neutrophil activa-

\section{Figure 6}

MHC I mAb-induced lung injury is characterized by an acute inflammatory and antiinflammatory response and an increase in plasma and BAL chemokines. (A) Plasma samples obtained 2 hours after anti-MHC I mAb treatment showed higher levels of inflammatory cytokines TNF- $\alpha$ and IL- 6 as well as elevated levels of IL-10 compared with controls ( $n=5$ per group). (B and C) $\mathrm{KC}$ and MIP-2 levels were also increased in the plasma (2 hours after MHC I mAb treatment) (B) and the BAL (C) of MHC I mAb-treated mice ( $n=6$ per group). ${ }^{*} P<0.01$. tion assay, we found there was no evidence of direct activation of neutrophil oxidative burst activity by the MHC I mAb in either wild-type or FcR $\gamma^{-1-}$ cells (Figure 10B).

MHC I mAb-mediated ALI is independent of C5a. Since clinical studies (21) and other animal models of TRALI (8) have implicated complement activation, we used $\mathrm{C} \mathrm{a} \mathrm{R}^{-/-}$mice to test the role of complement in the development of MHC I mAb-mediated ALI. When $\mathrm{C} \mathrm{aR}^{-/-}$mice on the $\mathrm{BALB} / \mathrm{c}$ background were given i.v. MHC I mAb, severe lung injury developed that was no different from lung injury in BALB/c wild-type controls (data not shown). Furthermore, there was approximately $66 \%$ mortality in the $\mathrm{C}^{\mathrm{a}} \mathrm{a \textrm {R } ^ { - / - }}$ mice, which was similar to the approximately $50 \%$ mortality observed in BALB/c wild-type mice (Figure 2A).

\section{Discussion}

The major findings of this study are that (a) the transfusion of an MHC I mAb to mice with the cognate antigen produced ALI and mortality in a clinically relevant time period; (b) the lung edema resulted from a combination of both increased lung vascular and lung epithelial permeability to protein and decreased alveolar fluid clearance, what we believe to be a novel finding in TRALI; and (c) neutrophils and the $\mathrm{Fc} \gamma$ receptors on neutrophils were essential to the pathogenesis of ALI in this mouse model of TRALI.

An important goal of this study was to develop a clinically relevant, reproducible, and robust model of human TRALI. There are several lines of evidence that suggest that this mouse model of TRALI relates well to the human condition. First, our model is based on the transfusion of MHC I Abs, the most frequently isolated $\mathrm{Ab}$ in fatal cases of TRALI reported to the FDA in 2001-2003 (6). In addition, there are numerous case reports and case series in the literature that report the association of MHC I Abs with fatal and nonfatal TRALI $(2,5)$. Second, we selected a 2-hour experimental end point to reflect a time point of injury that is clinically observed in the majority of TRALI cases. The National Heart, Lung, and Blood Institute Consensus definition of TRALI states that the lung injury develops during or within 6 hours of the completion of the blood product transfusion (1). In addition, TRALI most commonly develops within the first 30 minutes to 1 hour after the initiation of blood product transfusion $(1,2)$. Mice in
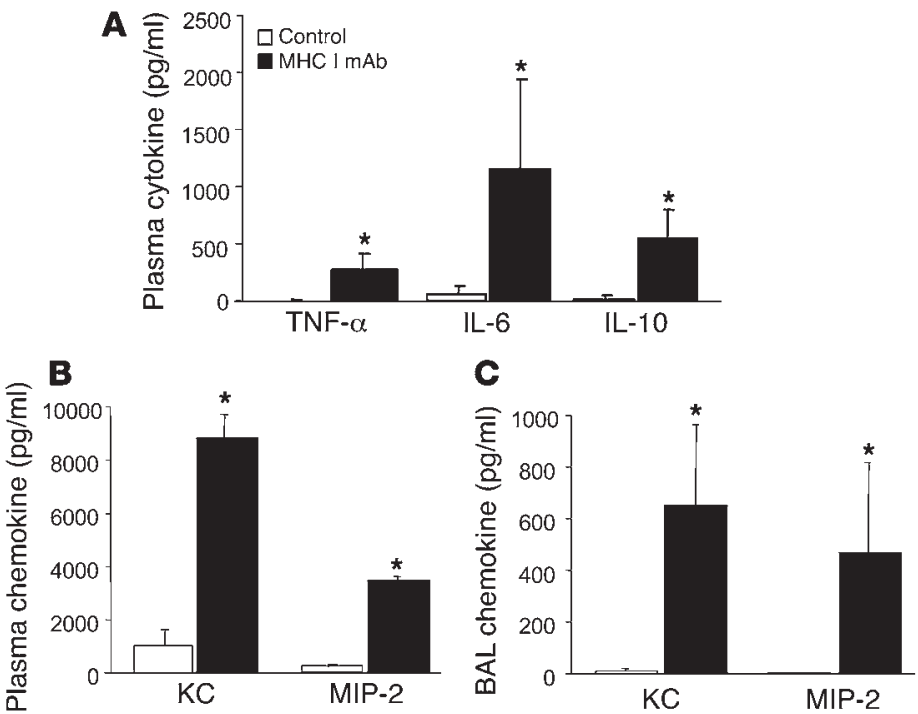


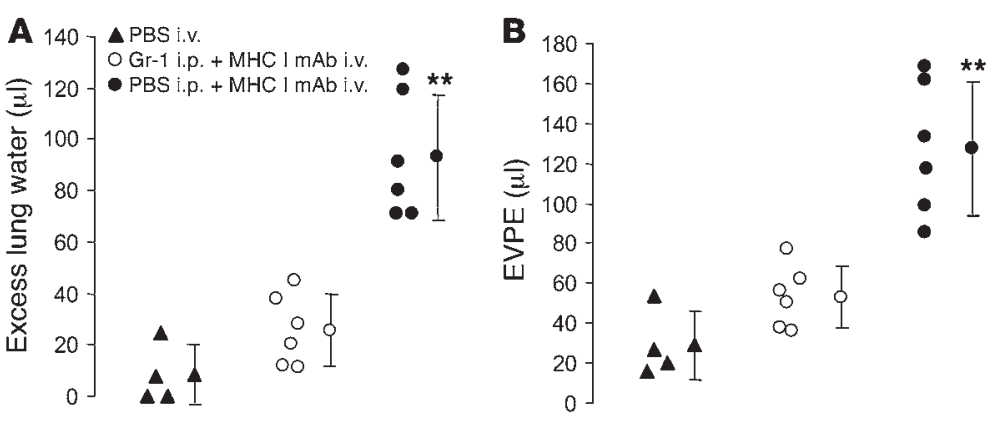

\section{Figure 7}

Neutrophil depletion with $\mathrm{Gr}-1$ mAb protects mice from MHC I mAb-induced ALI. Mice were pretreated with either i.p. PBS or i.p. Gr-1 mAb $(250 \mu \mathrm{g})$ and after 24 hours were given i.v. MHC I $\mathrm{mAb}$ and compared with mice given i.v. PBS. The Gr-1 mAb-pretreated mice had greatly attenuated excess lung water $(\mathbf{A})$ and lung vascular permeability to ${ }^{125}$-labeled albumin (B) compared with mice pretreated with PBS. Each data point represents an individual animal. ${ }^{* \star} P<0.01$. our model of TRALI developed lung injury within a 2-hour experimental time point, often within 30 minutes of MHC I mAb injection, and therefore this model is consistent with the time course of clinical TRALI.

Our experimental results of transfusing MHC I mAb to mice also reveal several features that model human TRALI well. First, the lung injury that developed was often severe, with 50\% mortality in these unsupported mice at 2 hours. The majority of human TRALI cases require intensive care support and mechanical ventilation, and there is $5-10 \%$ mortality (5). Second, the lung injury in our model was characterized by increased lung vascular permeability to protein, the defining characteristic of ALI. Yost (28) and others $(29,30)$ have reported that the undiluted pulmonary edema fluid of well-characterized patients with TRALI contains a high protein concentration consistent with increased permeability edema. Autopsy studies have also shown proteinaceous edema fluid in patients dying of TRALI (31). Third, we detected peripheral blood neutropenia in MHC I mAb-treated mice, as has been reported in human TRALI $(2,18)$. Finally, the histologic finding of extensive neutrophil sequestration in the lung microvasculature has also been reported in autopsy reports on TRALI (31).

We have also made what we believe to be novel findings in Abmediated TRALI. The existing ex vivo animal models of TRALI have focused on the lung endothelium and indirect measures of capillary permeability. In this MHC I mAb model of TRALI, not only was there increased permeability of the lung endothelium, but there was also a striking increase in lung epithelial permeability with a concomitant impairment in fluid resorptive capacity. We believe the finding that the alveolar epithelium was a target of damage in Ab-mediated TRALI to be novel and to have potential therapeutic implications. Research done by our group and other investigators has found that decreased alveolar fluid transport is a common finding in both experimental ALI (12) and in human patients with ALI and acute respiratory distress syndrome (ARDS) $(13,14)$. In fact, human patients with ALI/ARDS and impaired alveolar fluid clearance have higher mortality than do ALI/ARDS patients with intact alveolar fluid clearance. It is possible that the presence or absence of impaired alveolar fluid clearance in patients with TRALI could contribute to the clinical outcome in this syndrome - patients with more severe alveolar epithelial injury and impaired fluid clearance would likely have a worse prognosis.

We have made 2 fundamental observations about the pathogenesis of TRALI with this MHC I mAb model: the essential contribution of neutrophils and Fcy receptor signaling. The role of neutrophils in TRALI was first studied in an isolated, perfused rabbit lung model that used human neutrophils, a human antineutrophil Ab implicated in TRALI, and rabbit complement (8). The human neutrophils were essential to the development of lung injury in that model, but there was cross-species contamination. The peripheral blood neutropenia observed in our model and in several reported cases of TRALI also points to neutrophils potentially being sequestered in the lungs of TRALI patients, a hypothesis that was confirmed in our present model with histology and immunohistochemistry. Using 2 methods of neutrophil depletion, we now provide convincing evidence that neutrophils are the essential cellular effectors of lung injury in this mouse model of TRALI.

The factors leading to sequestration of neutrophils in the lung vasculature in TRALI are incompletely understood, but a 2-hit hypothesis has been offered to help explain the development of human and experimental TRALI (7). It is thought that underlying medical conditions (e.g., surgery and infection) prime circulating neutrophils and potentially lung endothelium, leading to lung neutrophil sequestration and an augmented response to a second challenge (e.g., blood products). The finding of increased

\section{Figure 8}

FcR $\gamma^{-1-}$ mice were protected from MHC I mAbinduced ALI and adoptive transfer of wild-type, and $B 2 \mathrm{~m}^{-/-}$neutrophils restored lung injury in $\mathrm{FCR} \gamma^{-/-}$ mice. $F c R \gamma^{-/-}$mice, wild-type matched controls (BALB/C), and $F c R \gamma^{-1-}$ mice injected with $5 \times 10^{6}$ wild-type or $\mathrm{B}^{-/-}$neutrophils were given i.v. $\mathrm{MHC}$ I $\mathrm{mAb}$, and excess lung water $(\mathbf{A})$ and lung vascular permeability (B) were measured. Each data point represents an individual animal. ${ }^{* \star} P<0.01$, $F_{C} R \gamma^{-1-}$ versus wild-type and $F_{C} R \gamma^{-1-}$ plus wild-type or $B 2 m^{-/-}$neutrophils.

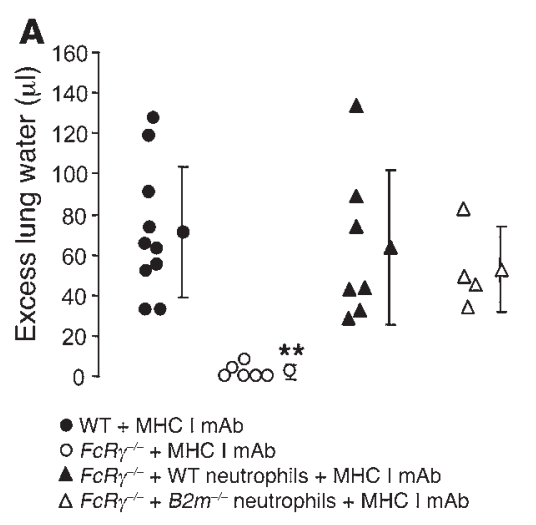

$\triangle F_{C} R^{-t}+$ WT neutrophils + MHC I mAb

$\triangle F_{C} R_{\gamma}+B 2 m$ neutrophils $+\mathrm{MHC} I \mathrm{mAb}$
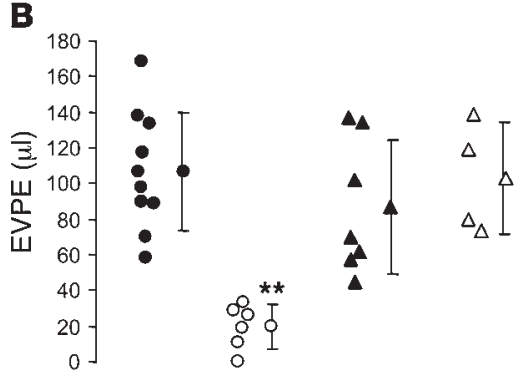

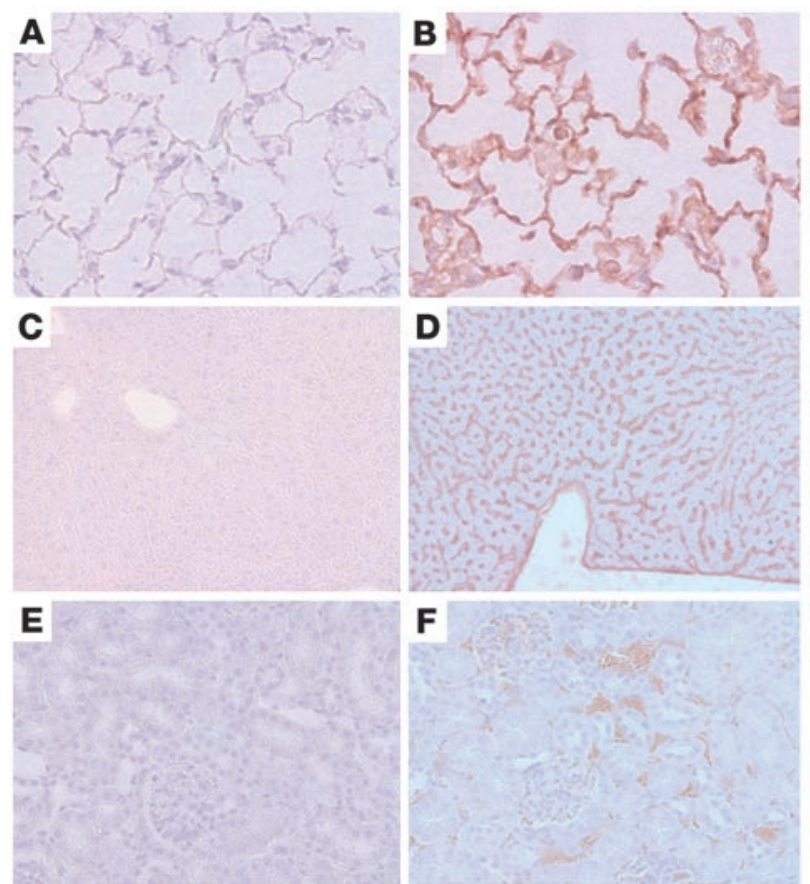

$\mathbf{F}$

Figure 9

Distribution of $\mathrm{MHC}$ I mAb after i.v. injection. Rag2 $^{-/-}$mice were injected i.v. with PBS (A, C, and E) or $4.5 \mathrm{mg} / \mathrm{kg} \mathrm{MHC} \mathrm{I} \mathrm{mAb}(\mathbf{B}, \mathbf{D}$, and $\mathbf{F})$ and sacrificed after 2 hours. The lungs (A and B; magnification, $\times 600$ ), liver (C and D; magnification, $\times 200)$, and kidney (E and F; magnification, $\times 400)$ were examined.

concentrations of human IL-8 homologs KC and MIP-2 in MHC I $\mathrm{mAb}$-induced TRALI adds to the understanding of lung neutrophil recruitment and sequestration. We hypothesize that neutrophils are mainly sequestered in the lung in this TRALI model from an $\mathrm{Fc}$ receptor interaction with endothelial-bound $\mathrm{MHC}$ I $\mathrm{mAb}$; however, additional recruitment of neutrophils by KC and MIP-2 may also be contributing.

The finding of increased plasma levels of TNF- $\alpha$ and IL- 6 in MHC I mAb-treated mice has also been reported in human TRALI patients and patients with ALI/ARDS, respectively. In a randomized controlled trial of fresh frozen plasma (FFP) transfusion in the intensive care unit, patients that received FFP from multiparous donors had a decrease in oxygenation and an increase in plasma levels of TNF- $\alpha$ (32). In a single patient who developed overt TRALI in that study, the plasma levels of TNF- $\alpha$ were markedly increased in the posttransfusion plasma. Plasma levels of IL-6 in patients with ALI/ARDS have been the most robust prognostic cytokine marker identified, with increased levels associated with increased mortality $(33,34)$.

We hypothesized that disease pathogenesis in our model was a result of immune recognition of MHC Ab bound to lung endothelium. To test this hypothesis, we challenged $F c R \gamma^{-/-}$mice with $\mathrm{MHC}$ I Ab. We found that mice that lack Fcy receptors were resistant to MHC I mAb-mediated lung injury. Neutrophil adoptive transfer experiments indicated that the $\mathrm{F} c \gamma$ receptors on the neutrophil specifically were especially important in TRALI pathogenesis. This contrasts with other Fcy receptor-dependent mouse models such as the $\mathrm{K} / \mathrm{BxN}$ arthritis model (35), immune complex peritonitis (36), and the Arthus reaction (37), in which
Fc $\gamma$ receptors on mast cells seem to be most important in initiating disease. Relevant to the lung, in a mouse model of alveolitis from intratracheal administration of immune complexes, lung injury was mast cell independent (38).

Of note, the protection observed in $F c R \gamma^{-/-}$mice argues against direct neutrophil activation by the MHC I mAb. FcR $\mathrm{\gamma}^{-/-}$mice possess the cognate antigen $\left(\mathrm{H} 2 \mathrm{~K}^{\mathrm{d}}\right)$, yet were protected from lung injury. We have also shown that direct neutrophil activation by MHC I mAb was absent in vitro (flow cytometry) and was not essential to restore lung injury in vivo $\left(B 2 \mathrm{~m}^{-/-}\right.$neutrophil transfer into $F c R \gamma^{-/}$mice). This contrasts with a recent TRALI model in which an anti-neutrophil mAb directly activated neutrophils in an ex vivo rat lung model (9). We hypothesize that the passively transfused MHC I mAb binds to MHC I antigen present on the pulmonary endothelium, the first vascular bed encountered after injection. The MHC I Ab bound to the lung endothelium sequesters circulating neutrophils via $\mathrm{Fc} \gamma$ receptor interaction, leading to neutrophil activation and lung injury. Elaboration of the neutrophil chemokines KC and MIP-2 probably also contributes to further neutrophil recruitment.

It was interesting to find that $\mathrm{C} 5 \mathrm{a}$ receptors were not essential in MHC I mAb-mediated ALI. Some animal models of immune complex alveolitis have shown that C5a is an essential chemokine in recruiting neutrophils into the lung and that it also directly activates neutrophils and endothelial cells $(39,40)$, although a recent animal model was complement independent (9). Future studies will be needed to completely address the role of complement in this TRALI model.

A limitation of our model of TRALI is that the use of mAbs may not exactly mimic the polyclonal MHC I, II, and anti-neutrophil Abs implicated in human TRALI. Another question in the TRALI literature is the dose or titer of Abs needed to produce TRALI in a recipient, although the presence of a priming condition and host susceptibility may determine this also. Indeed, it is possible that different anti-MHC I or -MHC II Abs would have different capacities to initiate neutrophil activation leading to lung injury. This is supported by our observation that not all anti-MHC I mAbs (all $\left.\operatorname{IgG}_{2 \mathrm{a}}, \kappa\right)$ we tested were able to induce ALI in BALB/c or C57BL/6J mice (data not shown). Hence in humans with different repertoires of polyclonal anti-MHC Abs, some Abs may be more pathogenic in transfused recipients than others. The issue of what is a pathogenic titer of anti-MHC Abs in a transfusion product will likely remain difficult to quantify.

A potential strength of this model of TRALI is the ability to examine other (non-lung) organ damage from MHC I Ab challenge. We found that our mAb bound diffusely to the liver and kidney microvasculature, and we have preliminary evidence of renal injury at our 2-hour endpoint. Nonpulmonary organ injury is an area that has been neglected in the human TRALI literature as well as in the existing TRALI models, which use isolated lung preparations. The presence or absence of major organ damage from challenge with an $\mathrm{Ab}$ against a ubiquitously expressed antigen could reveal new insights into the TRALI syndrome and will be a focus of future experiments.

In conclusion, this in vivo mouse model of TRALI based on MHC I $\mathrm{mAb}$ transfusion reproduces several features of human TRALI and helps overcome the limitations of existing TRALI animal models. Lung injury and death in this TRALI model resulted from a combination of lung vascular and lung epithelial permeability and the reduced ability of the alveolar epithelium to remove edema fluid. 
A
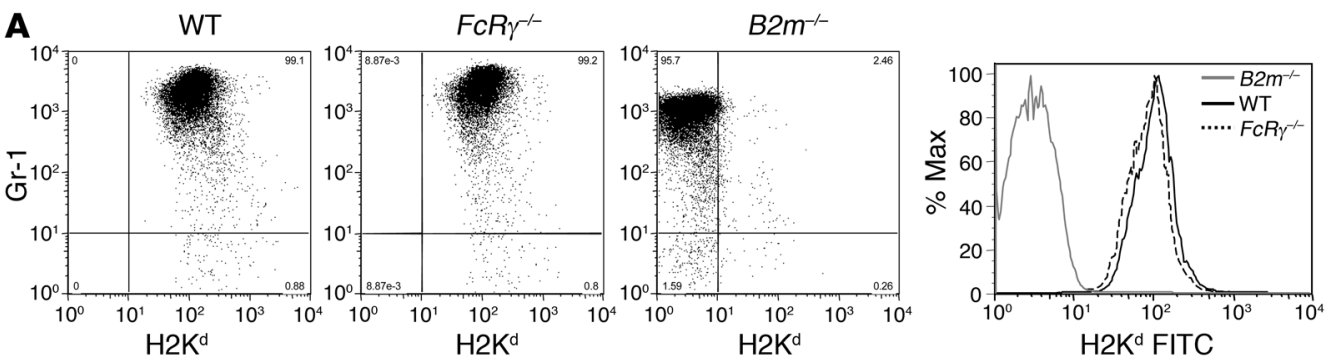

B

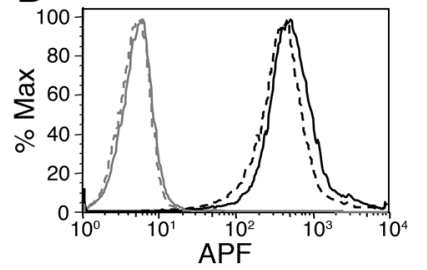

\section{Figure 10}

MHC I mAb binds but does not activate wild-type and $F C R \gamma^{-1-}$ neutrophils. (A) Flow cytometric analysis of MHC I expression on bone marrow neutrophils. Neutrophils were obtained from the bone marrow and stained with anti-H2Kd and the granulocyte-specific marker Gr-1. In $B 2 m^{-/-}$mice, the cells were negative for $\mathrm{H} 2 \mathrm{~K}^{\mathrm{d}}$ staining. (B) Flow cytometric analysis of superoxide production by bone marrow neutrophils. Superoxide anion levels were determined with an aminophenyl fluorescein (APF) flow cytometry assay. Mouse bone marrow neutrophils were either stimulated by $\mathrm{MHC}$ I cross-linking ( $25 \mathrm{mg} / \mathrm{ml}$ anti-H2 $\mathrm{K}^{\mathrm{d}}$-biotin followed by $40 \mathrm{mg} / \mathrm{ml}$ streptavidin) or $400 \mathrm{nM}$ PMA. Representative results from individual mice are shown.

Both the vascular endothelial and the alveolar epithelial injury were caused by neutrophil sequestration and activation in the lung through Fcy receptor interactions with endothelial-bound MHC I Ab. Additional inflammatory mediators produced during the response such as KC, MIP-2, TNF- $\alpha$, and IL- 6 further amplified the inflammation and neutrophil activation. Depletion of neutrophils protected animals from MHC I mAb-induced lung injury, while adoptive transfer experiments indicate that $\mathrm{Fc} \gamma$ receptors on neutrophils were the major receptors that initiated the inflammatory response. The results of these experimental studies suggest a potentially novel therapeutic strategy, targeting $\mathrm{Fc} \gamma$ receptor signaling pathways, that may prove beneficial in limiting lung injury in TRALI.

\section{Methods}

Experimental animals. BALB/c wild-type mice were purchased from Charles River Laboratories; BALB/c MHC I congenic mice (BALB/K) were purchased from The Jackson Laboratory, and $F c R \gamma^{-/-}$and $R a g 2^{-/-}$mice were from Taconic. C5aR $\mathrm{R}^{-/-}$and $\mathrm{B} 2 \mathrm{~m}^{-/-}$mice were kindly provided by C. Gerard (Children's Hospital Boston, Boston, Massachusetts, USA) and M. Anderson (University of California, San Francisco, San Francisco, USA), respectively. All experimental procedures were performed in 8- to 12-week-old male mice and were approved by the University of California, San Francisco Committee on Animal Research.

MHC I mAb production. A hybridoma (34-1-2S) was purchased from the American Type Culture Collection that produces a mAb against $\mathrm{H} 2 \mathrm{~K}^{\mathrm{d}}$ $\left(\mathrm{IgG}_{2 \mathrm{a}}, \mathrm{K}\right)$. The hybridoma was grown in tissue culture medium containing $1 \%$ fetal bovine serum and incubated at $37^{\circ} \mathrm{C}$ and $5 \% \mathrm{CO}_{2}$. Hybridoma supernatant was collected and filtered through a $0.2-\mu \mathrm{m}$ filter. The MHC I $\mathrm{mAb}$ was purified using protein A sepharose affinity chromatography and dialyzed overnight in PBS ( $\mathrm{pH}$ 7.4). The protein concentration of the $\mathrm{mAb}$ was spectrophotometrically determined using Bio-Rad protein reagent. The $\mathrm{mAb}$ solution $(0.5-0.65 \mathrm{mg} / \mathrm{ml})$ was frozen at $-80^{\circ} \mathrm{C}$ until the time of the experiments. For selected experiments, a protein biotinylation kit (Alpha Diagnostic International) was used to generate MHC I-biotin mAb.
Animal preparation and experimental protocol. For MHC I mAb injection, mice were anesthetized with i.p. ketamine $(80 \mathrm{mg} / \mathrm{kg})$ and xylazine $(12 \mathrm{mg} /$ $\mathrm{kg})$. The mice were placed supine on a warming blanket, and the jugular vein was isolated. Using a 30-gauge sterile needle attached to PE-10 tubing, venous blood was aspirated from the jugular vein to verify intravascular placement of the needle and to remove a sample of blood $(\sim 200 \mu \mathrm{l})$ for baseline measurements. Mice were given an i.v. volume-matched injection (150-250 $\mu \mathrm{l})$ of either MHC I mAb (4.5 mg/kg), an isotype-matched $\mathrm{mAb}$ ( $\mathrm{IgG}_{2 \mathrm{a}}, \kappa ; 4.5 \mathrm{mg} / \mathrm{kg}$; BD Biosciences - Pharmingen), or PBS. The skin was sutured with 6-0 silk suture, and the mice were recovered. Mice were euthanized at 2 hours with an i.p. injection of pentobarbital $(200 \mathrm{mg} / \mathrm{kg})$.

Measurement of excess lung water and lung vascular permeability. The gravimetric method was used to determine extravascular lung wet wt/dry wt ratios as previously described $(15,41)$. Briefly, the lungs were removed at the end of the experiment, and the wet weight was recorded. The lungs were then homogenized and dried for greater than 24 hours in a drying oven, at which time the dry weight was recorded. Measurements of the hemoglobin concentration in the lung homogenate allowed for the calculation of the bloodless lung wet wt/dry wt ratio.

For measurements of lung vascular permeability, ${ }^{125}$ I-labeled albumin (Iso-Tex Diagnostics Inc.) was added to the i.v. instillate. At the end of the experiment, the radioactivity of a blood sample and the whole lungs was determined with a gamma counter (Packard 5000 Series). The ratio of the radioactivity in the blood and in the bloodless lungs was used to calculate the lung vascular permeability to protein and the extravascular plasma equivalents (EVPE) as we have described previously $(15,23)$.

Alveolar epithelial permeability and BAL. Alveolar epithelial permeability was determined by the leakage of i.v. ${ }^{125}$ I-labeled albumin into the airspaces of the lung at the end of the experiment. A BAL was performed at the end of the experiment with $1 \mathrm{ml}$ of cold PBS flushed 3 times into the lung through a tracheotomy. The alveolar epithelial permeability was calculated by a ratio of the radioactivity/ $\mathrm{ml}$ in the BAL and in a blood sample (15). The total protein concentration in the BAL was determined by the Bradford method. 
Alveolar fluid clearance measurements. The in situ method for measuring alveolar fluid clearance was used as previously described $(42,43)$. Briefly, 2 hours after receiving either the MHC I mAb or control (isotype control $\mathrm{mAb}$ or PBS), mice were euthanized with i.p. pentobarbital $(200 \mathrm{mg} / \mathrm{kg})$ and exsanguinated by transaction of the abdominal aorta. Core temperature was maintained at $37-38^{\circ} \mathrm{C}$ (measured with a subdiaphragmatic temperature probe) with a warming blanket and infrared lamp. A tracheostomy was performed, and a 20-gauge trimmed angiocath was inserted into the trachea. The lungs were continuously inflated with $5 \mathrm{~cm} \mathrm{H}_{2} \mathrm{O}$ continuous positive airway pressure and $100 \% \mathrm{O}_{2}$. The trachea was instilled with $13 \mathrm{ml} / \mathrm{kg}$ of a $5 \%$ bovine serum albumin and lactated Ringer's solution. We also added $0.1 \mu \mathrm{Ci}{ }^{125} \mathrm{I}$-labeled albumin to the instillate as a radiotracer. One minute after the initial instillation, a sample was obtained from the distal airspaces to serve as the baseline sample. At 30 minutes another sample was obtained. The radioactivity of the 1-minute baseline and the 30-minute samples were determined with a gamma counter. The concentration of the ${ }^{125}$ I-labeled albumin at 30 minutes allowed for the calculation of alveolar fluid clearance.

Measurement of white blood cell count and differential in the blood. A sample of blood was placed in EDTA-coated vials, and a multispecies hematology instrument (Hemavet 950FS; Drew Scientific) was used to generate a complete blood count with cellular differential.

Neutrophil depletion experiments. Vinblastine ( $2.5 \mathrm{mg} / \mathrm{kg}$; Sigma-Aldrich) was given i.p. to mice, and neutrophil depletion (>95\%) was confirmed 72 hours later, before experiments with the MHC I mAb or control. A second method of neutrophil depletion was used with a rat anti-mouse $\mathrm{mAb}$ against the neutrophil maturation antigen, Gr-1 (BD Biosciences - Pharmingen). The Gr-1 mAb $(250 \mu \mathrm{g})$ was given i.p., and neutrophil depletion was confirmed 24 hours later.

Neutrophil isolation and reconstitution. Wild-type BALB/c mice were euthanized by cervical dislocation, and the bone marrow from the femurs and tibias was flushed with PBS using a 25-gauge needle. The whole bone marrow was centrifuged and washed in PBS, and the red blood cells were hypotonically lysed with $0.2 \% \mathrm{NaCl}$. This solution was resumed to isotonicity with $1.2 \% \mathrm{NaCl}$ and then filtered over a $70-\mu \mathrm{m}$ nylon cell strainer (BD Biosciences - Discovery Labware). The solution was centrifuged and resuspended in PBS and then delicately applied over a $62 \%$ Percoll gradient. The Percoll solution was centrifuged for 30 minutes at $1,500 \mathrm{~g}$. The neutrophil pellet was then isolated, washed and centrifuged twice, and counted with a Coulter counter (Z1 series; Beckman Coulter). Greater than $90 \%$ neutrophil purity was confirmed with a cytospin preparation (Cytospin 3; Thermo Electron Corp.) and Diff-Quick staining.

Multiplex cytokine and chemokine analysis. Mouse blood samples were collected in citrated syringes and centrifuged at $11,750 \mathrm{~g}$ for 10 minutes, and the plasma fraction was collected and frozen at $-80^{\circ} \mathrm{C}$. A mouse cytokine multiplex kit (Mouse Cytokine 10-plex; BioSource International) was used to assay for TNF- $\alpha$, IFN- $\gamma$, GM-CSF, IL-1 $\beta$, IL-2, IL-4, IL-5, IL-6, IL-10, and
IL-12. ELISA kits were used to measure murine KC and MIP-2 in the plasma and BAL (R\&D Systems).

Histology and immunohistochemistry. Mice were euthanized with i.p. pentobarbital, and a 20-gauge trimmed angiocath was inserted into the trachea. The lungs were then inflated with air and $4 \%$ paraformaldehyde. The trachea was ligated, and the lungs were immersed in $4 \%$ paraformaldehyde and embedded in paraffin. In selected experiments, the liver and the right kidney were also removed. Sections $(5 \mu \mathrm{m})$ were used for routine H\&E histology and for immunohistochemistry. Gr-1 mAb was used for neutrophil immunohistochemistry. For MHC I mAb immunohistochemistry, MHC I $\mathrm{mAb}$ was injected i.v. into Rag $2^{-/-}$mice, and the lungs were perfused with $\mathrm{PBS}$ via the right ventricle and processed as described above. MHC I mAb was detected in paraffin sections using a goat anti-mouse HRP detection kit (BD Biosciences).

Flow cytometry. Single-cell suspensions were prepared from wild-type, $F c R \gamma^{-/}$, and $\mathrm{B} \mathrm{m}^{-/-}$mouse bone marrow. Aliquots of $1.5 \times 10^{6}$ cells were stained with anti-MHC I-biotin $(1 \mu \mathrm{g} / \mathrm{ml})$ in the presence of Fc $\gamma \mathrm{RII} / \mathrm{III}-$ blocking $\mathrm{Ab}$ (2.4G2; BD Biosciences - Pharmingen) and anti-Gr-1-PE Ab (RB6-8C5; BD Biosciences - Pharmingen). Anti-MHC I-biotin was detected using streptavidin-FITC (BD Biosciences - Pharmingen). Neutrophils were gated by Gr-1 positivity and appropriate forward- and side-scatter settings. For in vitro ROS production, whole bone marrow was loaded with $10 \mu \mathrm{M}$ aminophenyl fluorescein (Invitrogen Corp.). MHC I was cross-linked by the addition of $25 \mu \mathrm{g}$ anti-MHC I-biotin in the presence of FcrRII/III-blocking Ab, followed by addition of $40 \mu \mathrm{g}$ streptavidin (Jackson ImmunoResearch Laboratories Inc.) and incubation at $37^{\circ} \mathrm{C}$ for 15,30 , and 60 minutes. ROS was induced by incubating neutrophils with $400 \mathrm{nM}$ PMA (Sigma-Aldrich) as a positive control. Flow cytometric acquisition was performed using FACScan (BD Biosciences), and data were analyzed using FlowJo software (version 6.4.3; Tree Star Inc.).

Statistics. Results are reported as individual data points and as mean $\pm \mathrm{SD}$. To determine significance, 2 -tailed Student's $t$ test, ANOVA, and $\chi^{2}$ test were used as appropriate. $P$ values less than or equal to 0.05 were determined to be significant.

\section{Acknowledgments}

This work was supported in part by a GlaxoSmithKline Pulmonary Fellowship Award (to M.R. Looney), by a Canadian Institutes of Health Research Doctoral Research Award (to J.A. Van Ziffle), and by NIH grants AI065495 (to C.A. Lowell) and HL51854 and HL81027 (to M.A. Matthay).

Received for publication October 27, 2005, and accepted in revised form April 4, 2006.

Address correspondence to: Mark R. Looney, 505 Parnassus Avenue, San Francisco, California 94143-0130, USA. Phone: (415) 476-1079; Fax: (415) 502-2126; E-mail: mark.looney@ucsf.edu.
1. Toy, P., et al. 2005. Transfusion-related acute lung injury: definition and review. Crit. Care Med. 33:721-726.

2. Looney, M.R., Gropper, M.A., and Matthay, M.A. 2004. Transfusion-related acute lung injury: a review. Chest. 126:249-258.

3. Kopko, P.M., Marshall, C.S., MacKenzie, M.R., Holland, P.V., and Popovsky, M.A. 2002. Transfusion-related acute lung injury: report of a clinical look-back investigation. JAMA. 287:1968-1971.

4. Kleinman, S., et al. 2004. Toward an understanding of transfusion-related acute lung injury: statement of a consensus panel. Transfusion. 44:1774-1789.

5. Popovsky, M.A., and Moore, S.B. 1985. Diagnostic and pathogenetic considerations in transfusionrelated acute lung injury. Transfusion. 25:573-577.
6. Holness, L., Knippen, M.A., Simmons, L., and Lachenbruch, P.A. 2004. Fatalities caused by TRALI. Transfus. Med. Rev. 18:184-188.

7. Silliman, C.C., et al. 1997. The association of biologically active lipids with the development of transfusion-related acute lung injury: a retrospective study. Transfusion. 37:719-726.

8. Seeger, W., et al. 1990. Reproduction of transfusion-related acute lung injury in an ex vivo lung model. Blood. 76:1438-1444.

9. Sachs, U.J., et al. 2006. Antibody-induced neutrophil activation as a trigger for transfusion-related acute lung injury in an ex vivo rat lung model. Blood. 107:1217-1219.

10. Silliman, C.C., et al. 1998. Plasma and lipids from stored packed red blood cells cause acute lung injury in an animal model. J. Clin. Invest. 101:1458-1467.

11. Silliman, C.C., et al. 2003. Plasma and lipids from stored platelets cause acute lung injury in an animal model. Transfusion. 43:633-640.

12. Matthay, M.A., Folkesson, H.G., and Clerici, C. 2002. Lung epithelial fluid transport and the resolution of pulmonary edema. Physiol. Rev. 82:569-600.

13. Matthay, M.A., and Wiener-Kronish, J.P. 1990. Intact epithelial barrier function is critical for the resolution of alveolar edema in humans. Am. Rev. Respir. Dis. 142:1250-1257.

14. Ware, L.B., and Matthay, M.A. 2001. Alveolar fluid clearance is impaired in the majority of patients with acute lung injury and the acute respiratory distress syndrome. Am. J. Respir. Crit. Care Med. 163:1376-1383 
15. Wiener-Kronish, J.P., Albertine, K.H., and Matthay, M.A. 1991. Differential responses of the endothelial and epithelial barriers of the lung in sheep to Escherichia coli endotoxin. J. Clin. Invest. 88:864-875.

16. Fang, X., et al. 2002. Novel role for CFTR in fluid absorption from the distal airspaces of the lung. J. Gen. Physiol. 119:199-207.

17. Fukuda, N., Folkesson, H.G., and Matthay, M.A. 2000. Relationship of interstitial fluid volume to alveolar fluid clearance in mice: ventilated vs. in situ studies. J. Appl. Physiol. 89:672-679.

18. Nakagawa, M., and Toy, P. 2004. Acute and transient decrease in neutrophil count in transfusionrelated acute lung injury: cases at one hospital. Transfusion. 44:1689-1694.

19. Ausley, M.B., Jr. 1987. Fatal transfusion reactions caused by donor antibodies to recipient leukocytes. Am. J. Forensic Med. Pathol. 8:287-290.

20. Leger, R., Palm, S., Wulf, H., Vosberg, A., and Neppert, J. 1999. Transfusion-related lung injury with leukopenic reaction caused by fresh frozen plasma containing anti-NB1. Anesthesiology. 91:1529-1532.

21. Yomtovian, R., et al. 1984. Severe pulmonary hypersensitivity associated with passive transfusion of a neutrophil-specific antibody. Lancet. 1:244-246.

22. Ware, L.B., and Matthay, M.A. 2000. The acute respiratory distress syndrome. N. Engl. J. Med. 342:1334-1349.

23. Folkesson, H.G., Matthay, M.A., Hebert, C.A., and Broaddus, V.C. 1995. Acid aspiration-induced lung injury in rabbits is mediated by interleukin-8dependent mechanisms. J. Clin. Invest. 96:107-116.

24. Modelska, K., et al. 1999. Inhibition of beta-adrenergic-dependent alveolar epithelial clearance by oxidant mechanisms after hemorrhagic shock. Am. J. Physiol. 276:L844-L857.

25. Muhs, B.E., Gagne, P., Plitas, G., Shaw, J.P., and Shamamian, P. 2004. Experimental hindlimb isch- emia leads to neutrophil-mediated increases in gastrocnemius MMP-2 and -9 activity: a potential mechanism for ischemia induced MMP activation. J. Surg. Res. 117:249-254.

26. Plitas, G., et al. 2003. Experimental hindlimb ischemia increases neutrophil-mediated matrix metalloproteinase activity: a potential mechanism for lung injury after limb ischemia. J. Am. Coll. Surg. 196:761-767.

27. Takai, T., Li, M., Sylvestre, D., Clynes, R., and Ravetch, J.V. 1994. FcR gamma chain deletion results in pleiotrophic effector cell defects. Cell. 76:519-529.

28. Yost, C.S., Matthay, M.A., and Gropper, M.A. 2001. Etiology of acute pulmonary edema during liver transplantation: a series of cases with analysis of the edema fluid. Chest. 119:219-223.

29. Culliford, A.T., Thomas, S., and Spencer, F.C. 1980. Fulminating noncardiogenic pulmonary edema. A newly recognized hazard during cardiac operations. J. Thorac. Cardiovasc. Surg. 80:868-875.

30. Hashim, S.W., Kay, H.R., Hammond, G.L., Kopf, G.S., and Geha, A.S. 1984. Noncardiogenic pulmonary edema after cardiopulmonary bypass. An anaphylactic reaction to fresh frozen plasma. Am. J. Surg. 147:560-564.

31. Dry, S.M., Bechard, K.M., Milford, E.L., Churchill, W.H., and Benjamin, R.J. 1999. The pathology of transfusion-related acute lung injury. Am. J. Clin. Pathol. 112:216-221.

32. Palfi, M., Berg, S., Ernerudh, J., and Berlin, G. 2001. A randomized controlled trial oftransfusion-related acute lung injury: is plasma from multiparous blood donors dangerous? Transfusion. 41:317-322.

33. Parsons, P.E., et al. 2005. Lower tidal volume ventilation and plasma cytokine markers of inflammation in patients with acute lung injury. Crit. Care Med. 33:1-6.
34. The Acute Respiratory Distress Syndrome Network. 2000. Ventilation with lower tidal volumes as compared with traditional tidal volumes for acute lung injury and the acute respiratory distress syndrome. N. Engl. J. Med. 342:1301-1308.

35. Lee, D.M., et al. 2002. Mast cells: a cellular link between autoantibodies and inflammatory arthritis. Science. 297:1689-1692.

36. Godau, J., et al. 2004. C5a initiates the inflammatory cascade in immune complex peritonitis. J. Immunol. 173:3437-3445.

37. Sylvestre, D.L., and Ravetch, J.V. 1996. A dominant role for mast cell Fc receptors in the Arthus reaction. Immunity. 5:387-390.

38. Baumann, U., et al. 2001. Distinct tissue site-specific requirements of mast cells and complement components $\mathrm{C} 3 / \mathrm{C} 5$ a receptor in IgG immune complex-induced injury of skin and lung. J. Immunol. 167:1022-1027.

39. Bozic, C.R., Lu, B., Hopken, U.E., Gerard, C., and Gerard, N.P. 1996. Neurogenic amplification of immune complex inflammation. Science. 273:1722-1725.

40. Mulligan, M.S., et al. 1996. Requirement and role of $\mathrm{C} 5 \mathrm{a}$ in acute lung inflammatory injury in rats. J. Clin. Invest. 98:503-512.

41. Pittet, J.F., Wiener-Kronish, J.P., McElroy, M.C., Folkesson, H.G., and Matthay, M.A. 1994. Stimulation of lung epithelial liquid clearance by endogenous release of catecholamines in septic shock in anesthetized rats. J. Clin. Invest. 94:663-671.

42. Ma, T., Fukuda, N., Song, Y., Matthay, M.A., and Verkman, A.S. 2000. Lung fluid transport in aquaporin-5 knockout mice. J. Clin. Invest. 105:93-100.

43. Rezaiguia, S., et al. 1997. Acute bacterial pneumonia in rats increases alveolar epithelial fluid clearance by a tumor necrosis factor-alpha-dependent mechanism. J. Clin. Invest. 99:325-335. 